\title{
Comparison of the Mechanical Properties of AL6061/Albite and AL6061/Graphite Metal Matrix Composites
}

\author{
A. Ramesh $^{1}$, J. N. Prakash ${ }^{1 *}$, A. S. Shiva Shankare Gowda ${ }^{2}$ and \\ Sonnappa Appaiah ${ }^{3}$
}

\author{
${ }^{1}$ Department of Mechanical Engineering, Alpha College of Engineering \\ Bangalore-562149, INDIA \\ ${ }^{2}$ Dr.M.G.R Educational and Research Institute, \\ Chennai-600 095, INDIA \\ ${ }^{3}$ Department of Industrial Engineering and Management, M.S.R.I.T, \\ Bangalore-560 075, INDIA \\ *Corresponding author's e-mail address: prakash_jnp_ace@yahoo.com
}

\begin{abstract}
The aim of this present investigation is to carry out a comparative study of the mechanical properties of AL6061/Albite composites containing albite $\left(\mathrm{NaAlSi}_{3} \mathrm{O}_{8}\right)$ particulates, which are naturally occurring plagioclase feldspar and AL6061/graphite particulate composites containing graphite particles. The reinforcing particulates in the MMC's vary from $0 \%$ to $4 \%$ by weight. The 'vortex method' of production was employed to fabricate the composites, in which the reinforcements were poured into the vortex created by stirring the molten metal by means of a mechanical agitator. The composites so produced were subjected to a series of tests.
\end{abstract}

The results of this study revealed that as the Albite particle content was increased, there were significant increases in the ultimate tensile strength, hardness and Young's modulus, accompanied by a reduction in its ductility. There was, however, only a very marginal increase in the compressive strength, where as in graphite reinforced composites as the graphite content was increased, there were significant reduction in hardness and monotonic increases in the ductility, ultimate tensile strength (UTS), compressive strength and Young's modulus of the composite, An attempt is made in the paper to provide explanations for these phenomena.

KEYWORDS: Aluminium alloys; Albite; Graphite; Composites; Mechanical properties 


\section{INTRODUCTION}

Metal matrix composites (MMC's) are increasingly becoming attractive materials for advanced aerospace applications because their properties can be tailored through the addition of selected reinforcements [1-2]. In particular, particulate reinforced MMC's have recently found special interest because of their specific strength and specific stiffness at room or elevated temperatures [3]. It is well known that the elastic properties of metal matrix composites are strongly influenced by micro structural parameters of the reinforcement such as shape, size, orientation, distribution and volume fraction [4].

Aluminium-based Metal Matrix Composites (MMCs) have received increasing attention in recent decades as engineering materials. The introduction of a ceramic material into a metal matrix produces a composite material that results in an attractive combination of physical and mechanical properties which cannot be obtained with monolithic alloys. There is an increasing need for knowledge about the processing techniques and mechanical behaviour of particulate MMCs in view of their rising production volumes and their wider commercial applications. Interest in particulate reinforced MMCs is mainly due to easy availability of particles and economic processing technique adopted for producing the particulate-reinforced MMCs. Aluminium alloy-based particulate-reinforced composites have a large potential for a number of engineering applications. Interest in reinforcing Al alloy matrices with ceramic particles is mainly due to the low density, low coefficient of thermal expansion and high strength of the reinforcements and also due to their wide availability. Among the various useful aluminium alloys, aluminium alloy 6061 is typically characterized by properties such as fluidity, castability, corrosion resistance and high strength-weight ratio. This alloy has been commonly used as a base metal for MMCs reinforced with a variety of fibres, particles and whiskers [5-7].

In recent years, considerable work has been done on graphite reinforced metal matrix composites which exhibit low friction, low wear rate and excellent antiseizing properties. The graphite in these composites presumably imparts improved tribological properties to the composites through the formation of a graphite-rich film on the tribo-surface which provides solid lubrication. Journal bearings made of graphite particle dispersed composites perform much better than conventional bearing alloys [8].

Graphite particles of size ranging from 50 to $200 \mu \mathrm{m}$ yield the best results [9]. Manufacturing automotive pistons out of graphite reinforced composites instead of other conventional materials resulted in a saving of 5-7\% on fuel and lubricating oil [10]. The presence of graphite in the matrix improves its oil spreadability over the contact surface, thus reducing the tendency to score or seize. Graphite, which consists of carbon atoms arranged in a layerlike structure, displays a very low coefficient of friction while sliding on another clean surface, thus suggesting that it can be used as solid lubricants [11]. Because of this solid lubricative property, graphite in the form of particles has a wide range of applications in composite materials which are used to make components requiring great wear resistance such as engine bearings, pistons, piston rings and cylinder liners [12]. Although fibre 
reinforcements lead to marked enhancement in its properties, composites using particulate or discontinuous reinforcement for high-volume applications are being increasingly sought. The most widely used reinforcements have been $\mathrm{SiC}$, alumina, graphite, $\mathrm{B}_{4} \mathrm{C}$ and $\mathrm{TiC}$. These have been used to achieve improvements in selected properties. Most of the published data pertain to the mechanical properties of particulate-reinforced MMCs deal with tensile properties while only a relatively small amount of data has been obtained dealing with compression properties, although it is generally known that the compressive strength of an MMC is invariably higher than its UTS. Such MMCs are quite brittle compared with monolithic materials and have values of percentage elongation typically less than 5 per cent. For unreinforced aluminium alloy 6061, Awerbuch et al. [13] have shown that the deformation in compression could be greater than 50 per cent for specimens having a length-diameter ratio L/D equal to unity. Such effects could also be responsible for the apparently high value of the compressive strength. Hence, in the present investigation, importance is also given to the compressive properties of the MMCs, together with the tensile properties such as the UTS, ductility, hardness and Young's modulus.

In the present investigation, aluminium alloy AL6061 was used as the matrix material. AL6061 alloy has the highest strength and ductility of the aluminium alloys with excellent machinability and good bearing and wear properties [14]. Most of the particulate reinforced metal matrix composites are produced by liquid metallurgy, sometimes known as the 'vortex method' [15], although many different processes for fabricating these cast composites are also available which have been reported by various researchers. In the present work, the 'vortex method' of producing AMC's, in which graphite and albite particulates have been used as the candidate reinforcements of particulate sizes ranging from 100 to $150 \mu \mathrm{m}$ and added to the vortex formed in the AL6061 melt above its liquidus temperature. Since the ductility, ultimate tensile strength (UTS), compressive strength, Young's modulus and hardness of the composite material are all vital properties of a structural material, the present investigation aims at studying these properties in the AL6061 alloy/particulate composites.

\section{EXPERIMENTAL}

\subsection{Preparation of Composites}

In the present investigation, the materials tested were MMCs based on aluminium alloy 6061 (containing 0.4 per cent $\mathrm{Mg}$ and 0.75 per cent $\mathrm{Si}$ ) and containing graphite particulates of size 50 microns. The AL6061-graphite composites were prepared by the vortex method [9]. The graphite contents used for the preparation of the composites were $0 \%, 1 \%, 2 \%$ and $4 \%$. This is because graphite compositions of $7 \%$ and above would lead to rejection from the melt [16]. Addition of graphite into the molten aluminium alloy melt above its liquidus temperature of $500^{\circ} \mathrm{C}$ was carried out by creating a vortex in the melt using a mechanical stainless steel stirrer coated with aluminite (to prevent migration of ferrous ions from the stirrer material into the aluminium alloy melt). The melt was rotated at a speed of $500 \mathrm{rpm}$ in order to create the necessary vortex. The graphite particles were preheated to $400^{\circ} \mathrm{C}$ and added to the melt through the vortex at the rate of $0.1 \mathrm{~kg} / \mathrm{min}$. A small amount of magnesium, 
which improves the wettability of the graphite particles, was added along with the graphite, and the melt was thoroughly stirred and subsequently degassed by passing nitrogen through at the rate of $2-31 / \mathrm{min}$. The molten metal was then poured into permanent moulds for casting.

AL6061-Albite composites were produced using the same technique as used for graphite reinforced composites. The reinforcement used was albite $\left(\mathrm{NaAlSi}_{3} \mathrm{O}_{8}\right)$ particulates of diameter $90-150 \mu \mathrm{m}$, which is naturally occurring plagioclase feldspar. Basically consisting of silicates, it is abundantly available in the Earth's crust. Albite ranges from white to dark grey in colour and is extremely wear resistant, having a Moh hardness of about 6.5, almost rivaling that of $\mathrm{SiC}$ but exceeding that of alumina. Its specific gravity is about 2.6, which is much lower than those of $\mathrm{SiC}$ (3.1) and alumina (4.0). It does not react with the matrix material in this case, even at elevated temperatures, and has a low coefficient of thermal expansion of $2.3 \times 10^{-6} \mathrm{~K}^{-1}$. Such properties, coupled with its relative lightness, make albite a superior substitute for alumina and $\mathrm{SiC}$ as reinforcement material in MMCs. In the present work, the weight fraction of albite particulates used for making the MMCs was in the range of 0-4 per cent. In this technique, the albite particulates were slowly added into the vortex created in the molten aluminium alloy 6061 by stirring it with an impeller at $500 \mathrm{rpm}$. The melt containing the reinforcement was then poured in to permanent moulds for casting. The unreinforced matrix material was cast in the same manner, except that no albite particulates were added. The aluminium-alloy based composites containing various albite and graphite contents, namely 1, 2, 3 and $4 \mathrm{wt} \%$, were fabricated and tested, and their properties were compared with those of the unreinforced matrix. The tensile fracture specimens (Figures 3 and 4) were investigated by scanning electron microscopy (SEM) using a JEOL JSM 5410 instrument

\subsection{Testing of Specimens}

All tests were conducted in accordance with ASTM standards. Tensile tests were conducted at room temperature using a universal testing machine (UTM) in accordance with ASTM Standard E 8-82. The tensile specimens of diameter $8.0 \mathrm{~mm}$ and gauge length $75 \mathrm{~mm}$ were machined from the cast composites with the gauge length of the specimens parallel to the longitudinal axis of the castings. For each composite, four tensile test specimens were tested and the average values of the UTS, Young's modulus and ductility (in terms of percentage elongation) were measured. The hardness tests were conducted in accordance with ASTM Standard E 10 using a Brinell hardness tester with a ball indenter of $2.5 \mathrm{~mm}$ diameter and a load of $31.25 \mathrm{~kg}$. The load was applied for 30 secs. Eight hardness readings were taken for each specimen at different locations to circumvent the possible effects of particle segregation. Compression tests were conducted on a UTM in accordance with ASTM Standard E 9 at room temperature. In this test the compression loads were gradually increased and the corresponding strain was measured until the specimen failed. Each result is an average of four readings. 


\section{RESULTS AND DISCUSSIONS}

Table 1 and 2 shows the results for ductility (in terms of percentage elongation), ultimate tensile strength (UTS), compressive strength, hardness and Young's modulus obtained for the composites containing various amounts of graphite and albite. Each value represented is an average of six measurements. The results are repeatable in the sense that each individual result did not vary more than $5 \%$ from the mean value.

All these results are represented graphically in Figures 4-14. The optical micrographs Figure 1 and Figure 2 shows the graphite reinforced AL6061/Graphite composite and Albite reinforced AL6061/Albite composite at low and higher magnifications. The microstructures of the composites shown differ only in the degree of reinforcing particulates clustering; localized enrichment of particulates is visible in the matrix.
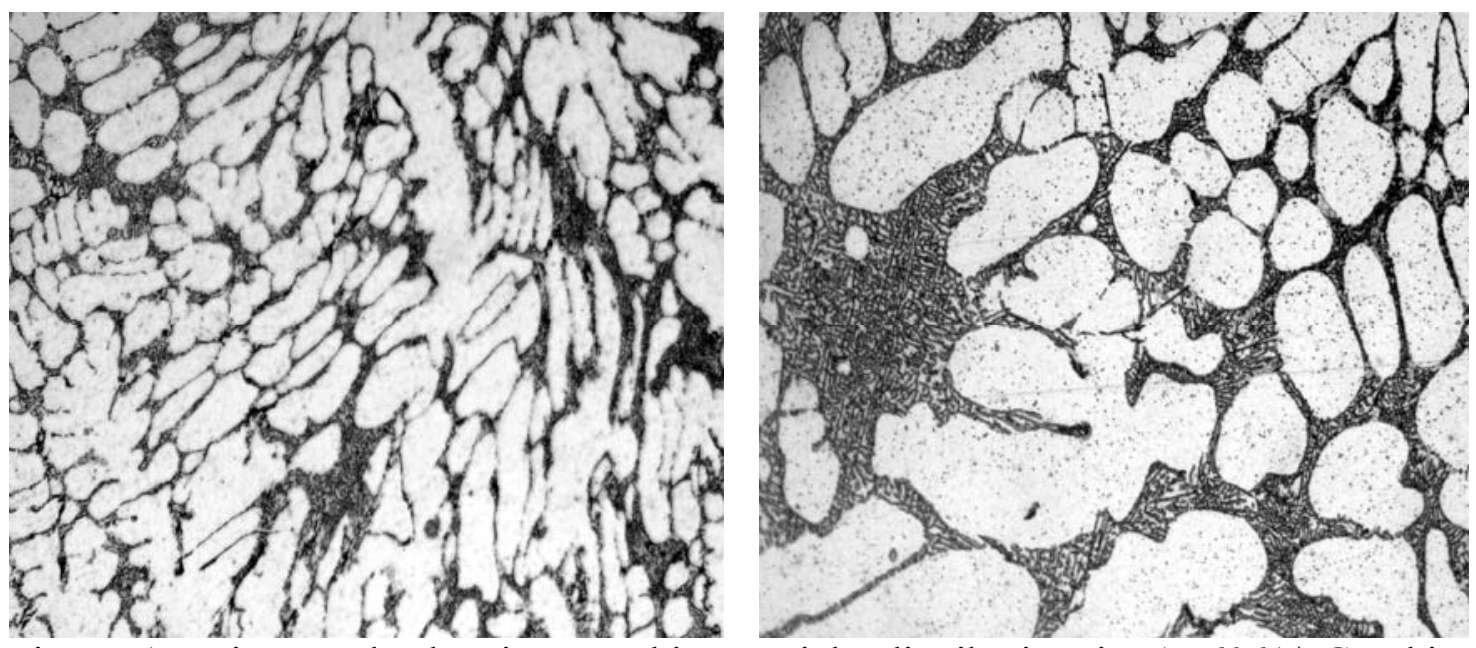

Figure 1. Micrograph showing graphite particle distribution in AL6061/ Graphite composites
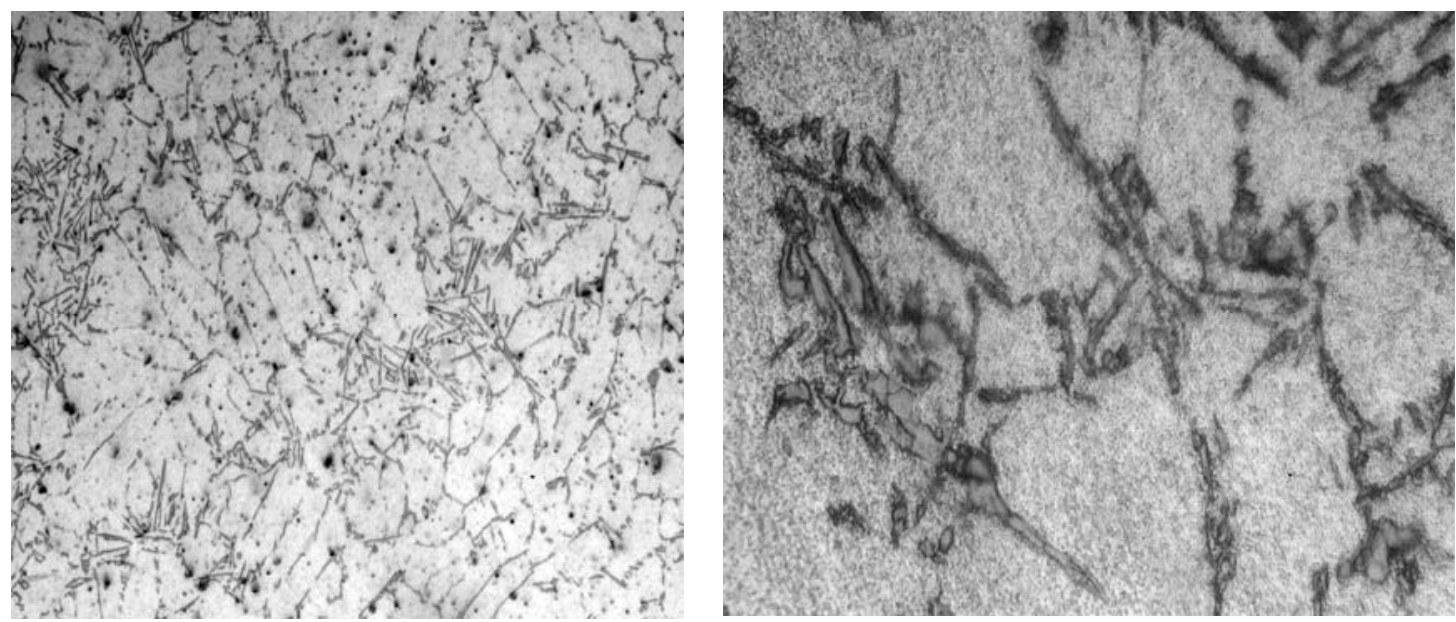

Figure 2. Micrograph showing Albite particle distribution in AL6061/ Albite composites 


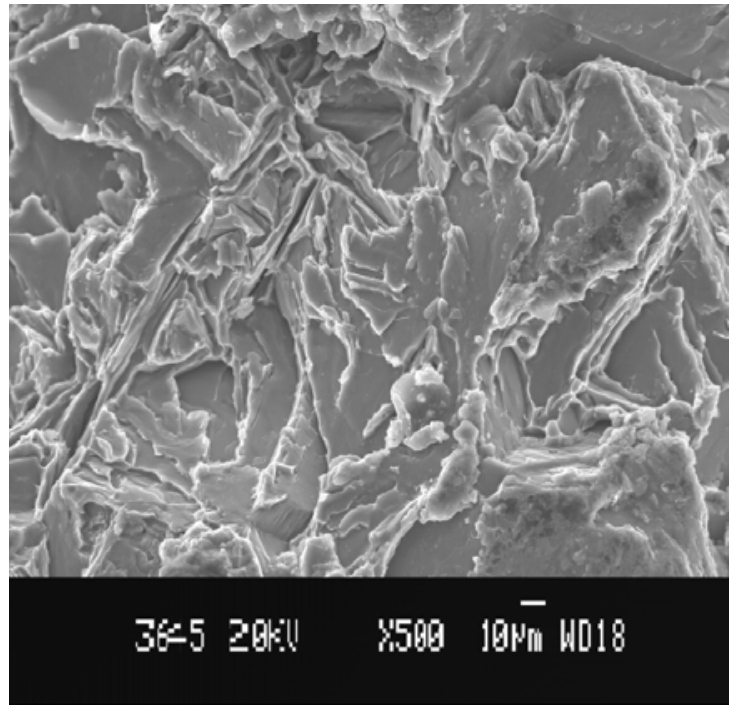

Figure 3. Tensile Fractured SEM image of 4\% by weight Graphite reinforced AL6061 based MMC

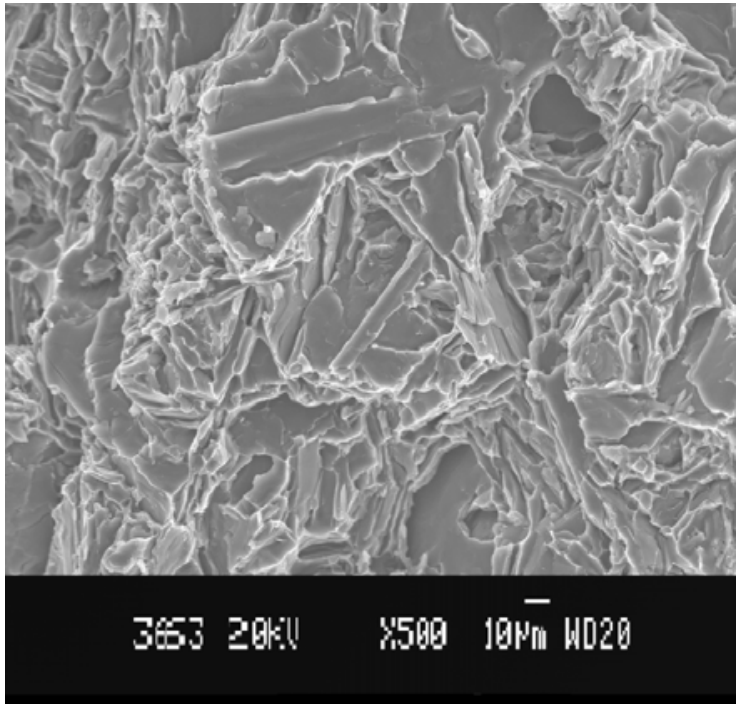

Figure 4. Tensile Fractured SEM image of 4\% by weight Albite reinforced AL6061 based MMC

\subsection{Ultimate Tensile Strength}

Figure 5 is a graph showing the effect of reinforcement content on the UTS of cast AL6061/graphite and AL6061/Albite particulate composites. It can be seen that as the graphite content increases, the UTS of the composite material increases monotonically by significant amounts. In fact, as the graphite content is increased from $0 \%$ to $4 \%$, the UTS increases by about $25 \%$. These results are in accordance with those obtained by Pillai [17] who reported similar findings. This increase in UTS may be due to the graphite particulates acting as barriers to dislocations in the microstructure [18]. One great advantage of this dispersion-strengthening effect is that it is retained even at elevated temperatures and for extended time periods because the particles are unreactive with the matrix phase [19].

\section{Table 1: Mechanical Properties of AL6061-Graphite composites containing varying} amounts of Graphite

\begin{tabular}{cccccc}
\hline $\begin{array}{l}\text { Graphite } \\
\text { particulate } \\
\begin{array}{l}\text { content } \\
(\%)\end{array}\end{array}$ & UTSMpa & $\begin{array}{l}\text { Brinell } \\
\text { Hardness } \\
\text { (HB) }\end{array}$ & $\begin{array}{l}\text { Young's } \\
\text { modulus } \\
\text { Gpa }\end{array}$ & $\begin{array}{l}\text { Ductility(\% } \\
\text { elongation) }\end{array}$ & $\begin{array}{l}\text { Ultimate } \\
\text { Compression } \\
\text { Strength } \\
\text { (Mpa) }\end{array}$ \\
\hline 0 & 153.90 & 51.41 & 71.47 & 5.90 & 743.03 \\
1 & 173.80 & 44.58 & 73.65 & 7.31 & 847.90 \\
2 & 181.20 & 40.64 & 76.34 & 8.47 & 946.10 \\
3 & 182.10 & 39.98 & 77.10 & 8.60 & 1028.72 \\
4 & 191.65 & 37.38 & 78.02 & 9.40 & 1067.21 \\
\hline
\end{tabular}

Figure 5 also shows the effect of the albite particulate content on the UTS of the composites. It can be seen that the UTS of the composite increases monotonically by over 30 per cent as the albite particulate content is increased from 0 to $4 \mathrm{wt} \%$. The increase in UTS can be attributed to the presence of hard albite particulates that impart strength to the matrix alloy, thereby providing enhanced resistance to tensile stresses. There is a reduction in the interspatial distance between the hard albite particulates, which causes an increase in the dislocation pile-up as the particulate content is increased. This leads to a restriction in the 
plastic flow due to the random distribution of the particulates in the matrix, thereby providing enhanced tensile strength to the composites. Vogelsang et al. [5], believed that the improvement in the UTS may be due to the matrix strengthening following a reduction in composite grain size, and the generation of a high dislocation density in the matrix as a result of the difference between the thermal expansion coefficients of the metal matrix and the albite particulate reinforcement.

\section{Table 2: Mechanical Properties of AL6061-Albite composites containing varying amounts of Albite}

\begin{tabular}{lcllcc}
\hline $\begin{array}{l}\text { Albite } \\
\text { particulate } \\
\text { content } \\
(\%)\end{array}$ & UTSMpa & $\begin{array}{l}\text { Brinell } \\
\text { Hardness } \\
\text { (HB) }\end{array}$ & $\begin{array}{l}\text { Young's } \\
\text { modulus } \\
\text { Gpa }\end{array}$ & $\begin{array}{l}\text { Ductility(\% } \\
\text { elongation) }\end{array}$ & $\begin{array}{l}\text { Ultimate } \\
\text { Compression } \\
\text { Strength } \\
\text { (Mpa) }\end{array}$ \\
\hline \multicolumn{1}{c}{0} & 153.9 & 51.41 & 71.47 & 5.90 & 743.03 \\
1 & 178.17 & 72.22 & 80.71 & 5.25 & 753.41 \\
2 & 182.34 & 73.57 & 85.47 & 4.57 & 757.5 \\
3 & 184.21 & 75.60 & 88.00 & 4.10 & 767.86 \\
4 & 201.34 & 99.10 & 99.95 & 3.95 & 780.01 \\
\hline
\end{tabular}

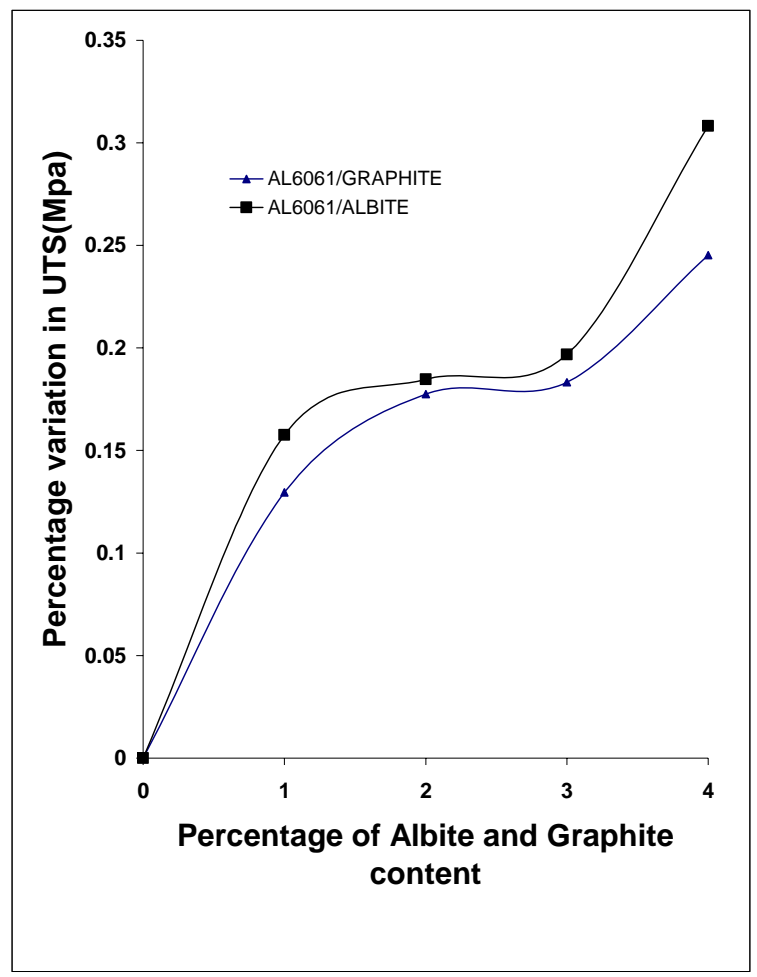

Figure 5. Effect of the albite and graphite content on UTS.

Similar observations were made by Ghosh and Ray [20], who fabricated $\mathrm{Al}_{2} \mathrm{O}_{3}$ particulatereinforced aluminium alloy composites using the compo-casting method and by McCoy et al. [21] who produced $\mathrm{TiB}_{2}$-particulate-reinforced aluminium alloy composites with particulate contents ranging from 10 to $25 \mathrm{vol} \%$. An increase in the UTS was observed as the $\mathrm{TiB}_{2}$ 
content was increased from 10 to 18 vol \%. One significant advantage of this dispersionstrengthening effect is that it is retained even at elevated temperatures and for extended periods of time because the albite particulates do not react with the matrix phase [19]. It is noteworthy that the most spectacular increase in the UTS occurs when the albite particulate content is increased from 0 to $1 \mathrm{wt} \%$. Quantitatively, this increase in the UTS is over 15 per cent. Subsequent equivalent additions of albite particulates, however, do not result in such great increases in the UTS. The bar graph (Fig. 6) shows the comparative changes that the albite and graphite reinforcements have on the tensile strength on the matrix alloy AL6061.

\subsection{Hardness}

Hardness, which is described as a measure of a material's resistance to surface indentation, may be thought of as a function of the stress required to produce some specific types of surface deformation. Figure 7 is a graph showing the effect of graphite and albite reinforcements on the hardness of cast AL6061/graphite and AL6061/Albite particulate composites. It can be seen that as the graphite content increases, the hardness of the composite material decreases monotonically by significant amounts. In fact, as the graphite content is increased from $0 \%$ to $4 \%$ the hardness decreases by about $27 \%$. One would have expected that as the UTS of a material increases, so would the hardness, as is very evident for the common engineering metals like steel, and indeed for many composites too. Nevertheless, the opposite is seen in this particular composite, whereby the hardness drops as the UTS increases. There is a good reason for this phenomenon, though, since graphite, being a soft dispersoid, does not contribute positively to the hardness of the composite. Instead, as mentioned above in the case of ductility, the graphite added, being an effective solid lubricant [22,8-11], eases the movement of grains along the slip planes, rendering the material more easily deformable under the indenter of the hardness tester. K.H.W Seah et al., [23] have reported a reduction in hardness from $107 \mathrm{BHN}$ to $77 \mathrm{BHN}$ (about 28\% differences) on addition of similar weight percentages of graphite to ZA-27 (Zinc Aluminium) alloy. Such a monotonic decrease in the hardness of the composite as graphite content is increased poses a limit to how much graphite may be added to enhance its other mechanical properties, since hardness is directly related to wear resistance. Consequently, a compromise is necessary when deciding how much graphite should be added to enhance the ductility, UTS, compressive strength, and Young's modulus of the composite without sacrificing too much of its hardness, especially in components like engine bearings, pistons, piston rings and cylinder liners, in which wear resistance is of paramount importance.

Figure 7 is a graph showing the effect of percentage in hardness, with increase in albite particulate content on the hardness of the composites. It is evident that, as the percentage of albite particulates is increased from 0 to $4 \mathrm{wt} \%$, the hardness of the composite increases monotonically and significantly to almost twice its original value. Zhu and Liu [24] also observed a similar increase in hardness when ZA alloy is reinforced with short alumina fibres. Various other researchers have also reported that the addition of hard ceramic particulates or short fibres to metal alloys could lead to improved strength, wear resistance and hardness [6-7]. A similar effect was observed by Sood et al. [25] for TiC reinforced aluminium alloy MMCs. They found that the hardness linearly increases with increasing volume percentage of $\mathrm{TiC}$. The increase in hardness is to be expected since it is observed that the increased UTS of most engineering materials such as steel and many composites lead to an increased hardness. Albite particulates, being hard (6 on the Moh scale), exhibit a greater resistance to indentation by the hardness tester and hence enhanced hardness since the hardness, after all, it is a measure of the resistance of a material to surface indentation and is a 
function of the stress required to produce some specific type of surface deformation [26]. The increased hardness is also attributed to the fact that the hard albite particulates act as barriers to the movement of the dislocations within the matrix.

As in the case of the UTS described above, the most spectacular increase in the hardness occurs when the albite particulate content is increased from 0 to $1 \mathrm{wt} \%$. Quantitatively, this increase in hardness is more than 40 per cent. Subsequent equivalent additions of albite particulates, however, do not result in such great increases in hardness. The bar graph (Fig 8) shows the comparative changes in hardness values, the albite and graphite reinforcements have on the on the matrix alloy AL6061.

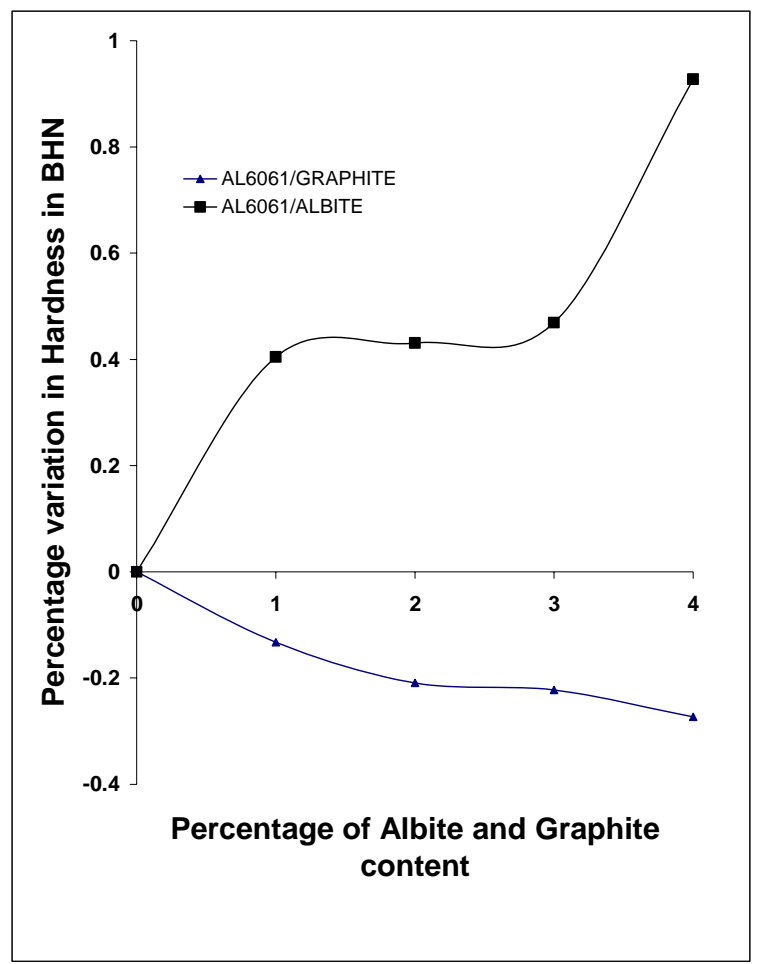

Figure 7. Effect of the albite and graphite content on Hardness

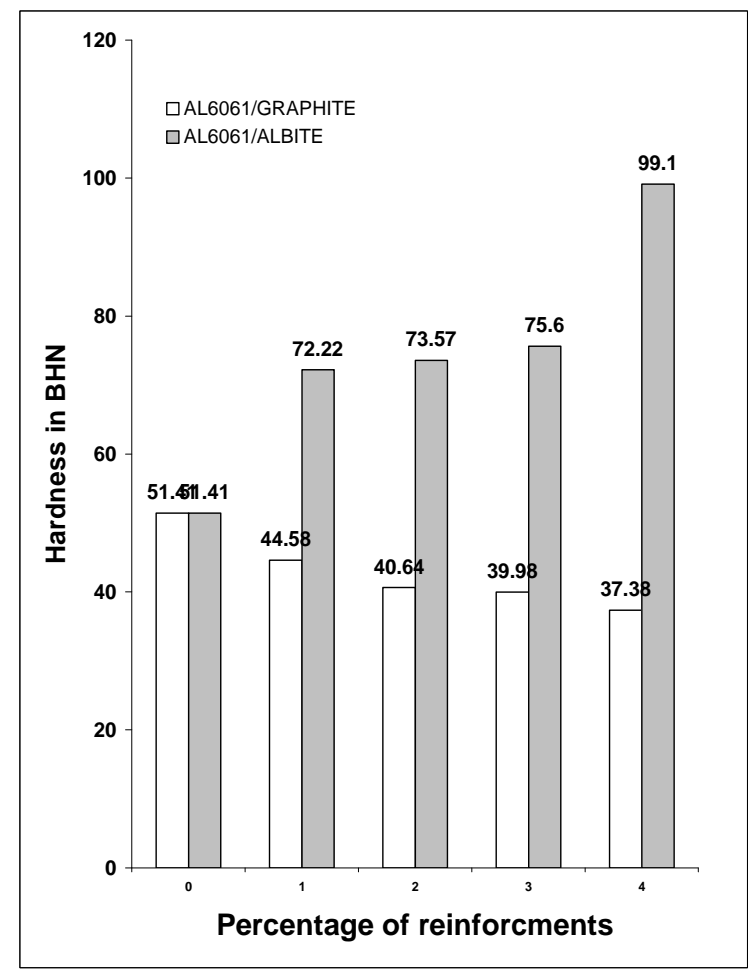

Figure 8. Bar graph of hardness values of AL6061/Graphite and AL6061/Albite metal matrix composites.

\subsection{Young's Modulus}

Figure 9 is a graph showing the percentage variation in Young's modulus and the bar graph (Figure 10) provides a comparative insight in to the actual experimental results recorded for the AL6061 albite and graphite particulate reinforced composites. The effect of graphite content on the Young's modulus of cast AL6061/graphite particulate composites. As in the cases of ductility, UTS and compressive strength described above, it can be seen that as the graphite content increases, the Young's modulus of the composite material increases monotonically by significant amounts. In fact, as the graphite content is increased from $0 \%$ to $4 \%$, the Young's modulus increases by about $9 \%$. As in the cases of UTS and compressive strength described above, this increase in compressive strength may be due to the graphite particles acting as barriers to dislocations in the microstructure [18]. Similar results have been obtained in aluminium matrix composites where the Young's modulus has been reported to 
increase with increase in the content of the reinforcing material, regardless of the type of reinforcement used [27]. Nevertheless, it must be emphasized at this point that the values of Young's modulus presented in this paper are only useful for the purpose of comparison among themselves.

Figure 9 is a graph showing the effect of albite particulate content on Young's modulus of cast AL6061-albite particulate composites. As in the cases of the UTS and hardness described above, it can be seen that, as the albite particulate content increases, Young's modulus of the composite material increases monotonically and significantly by about 40 per cent as the reinforcement content is increased from 0 to $4 \mathrm{wt} \%$. This increase in Young's modulus is broadly in line with the rule-of-mixtures prediction. McDanels [27] who obtained similar results for particle-reinforced aluminium composites reported that Young's modulus increases with increase in reinforcement content regardless of the type of reinforcement used.

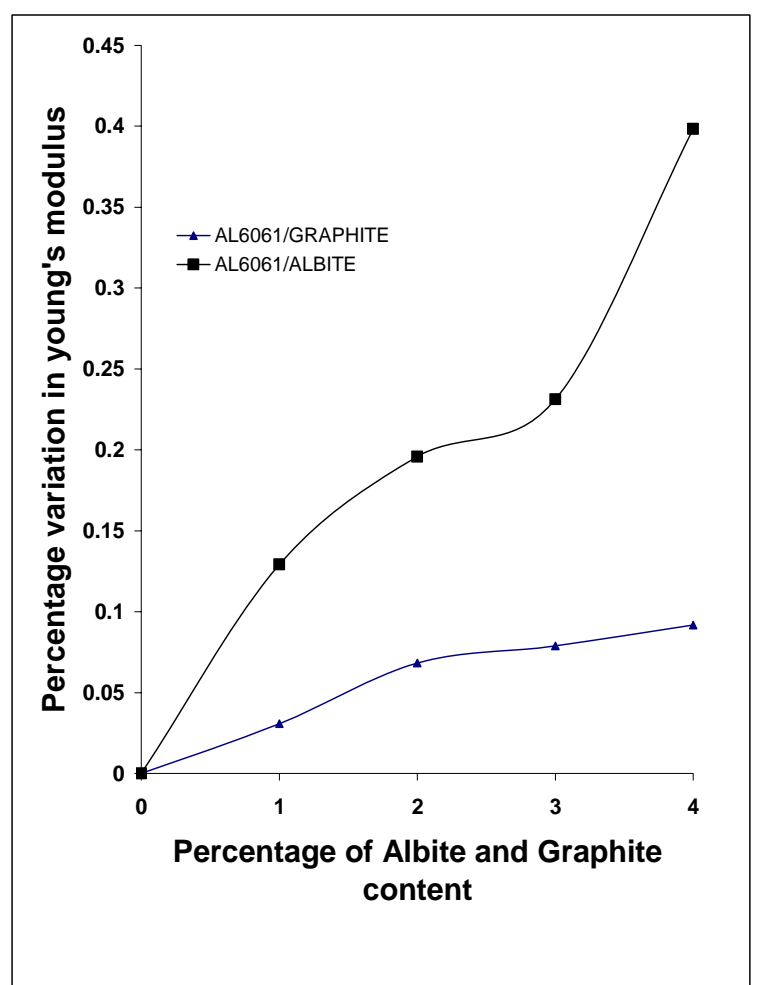

Figure 9. Effect of the albite and graphite content on Young's Modulus

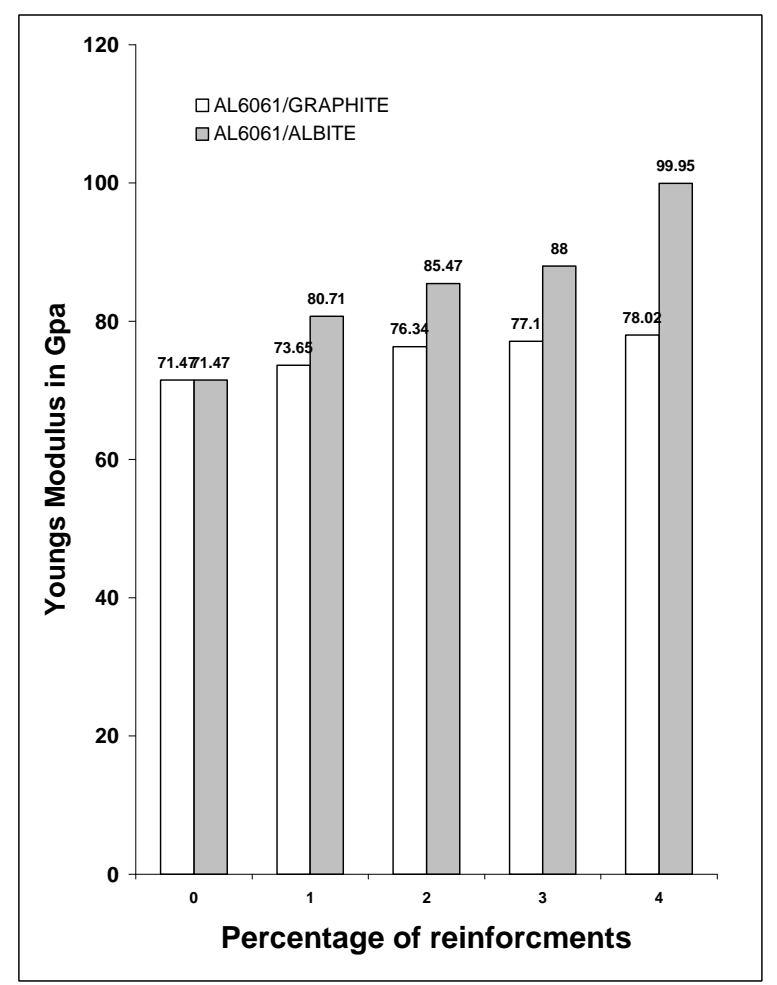

Figure 10. Bar graph of hardness values of AL6061/Graphite and AL6061/Albite metal matrix composites.

\subsection{Ductility}

Figure 11 is a graph showing the effect of graphite content on the ductility of cast AL6061/graphite particulate composites. It can be seen that, as in the case of UTS described above as the graphite content increases, the ductility of the composite material increases monotonically by significant amounts. In fact, as the graphite content is increased from $0 \%$ to $4 \%$ the ductility increases by about $60 \%$. This considerable increase in ductility is due to the graphite additions, being an effective solid lubricant [22, 8-11], eases the movement of grains along the slip planes. The effect of graphite is expected to be mechanical in nature since the particles are unreactive with the matrix phase [19]. 
Figure 11 is a graph showing the effect of albite particulate content on the ductility (measured in terms of percentage elongation) of the composites. It can be seen from the graph that the ductility of the composites decreases monotonically and significantly with the increase in albite particulate content. The ductility drops by about one-third as the albite particulate content is increased from 0 to $4 \mathrm{wt} \%$. These results tally with those obtained by other researchers $[29,24]$ who also observed that the ductility of the composites decreases with increase in the reinforcement content. This decrease in ductility in comparison with the matrix alloy is a most commonly encountered disadvantage in discontinuously reinforced MMCs [30].

The reduction in ductility can be attributed to the presence of a hard ceramic phase that is prone to localized crack initiation and increased embrittlement effect due to local stress concentration sites at the reinforcement-matrix interface. Hence, the introduction of this hard secondary ceramic phase creates slip regions. Moreover, the reinforcing particulates resist the passage of dislocations either by creating stress fields in the matrix or by inducing large differences in the elastic behaviour between the matrix and the dispersoid. The bar graph (Fig 12 ) shows the comparative changes in ductility, the albite and graphite reinforcements have on the on the matrix alloy AL6061.

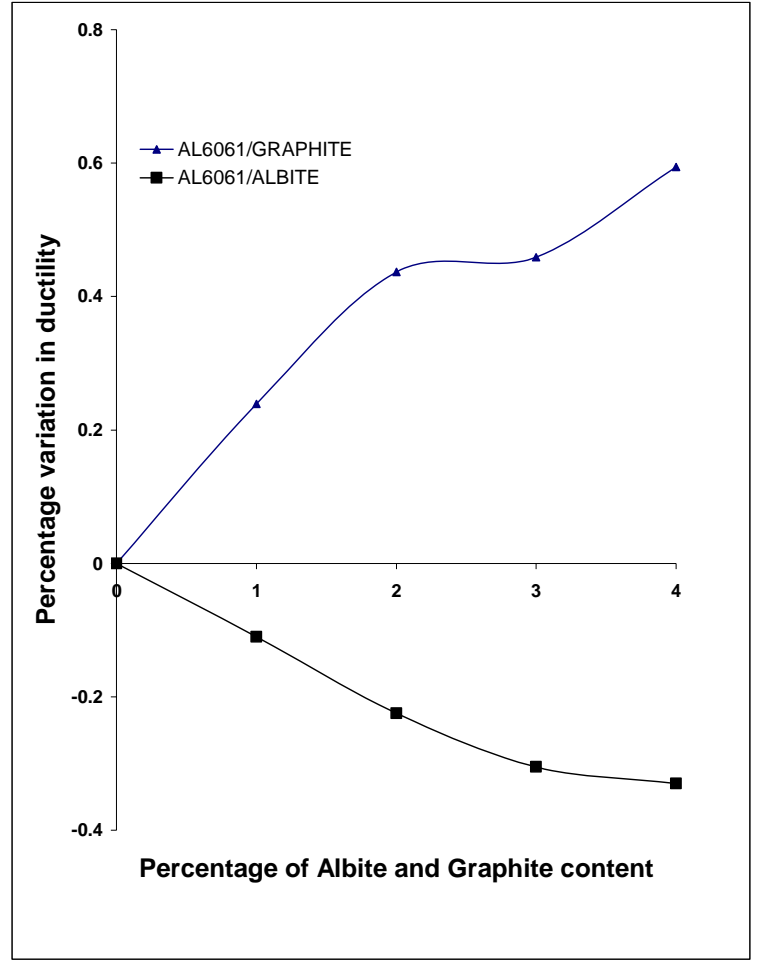

Figure 11. Effect of the albite and graphite content on Ductility

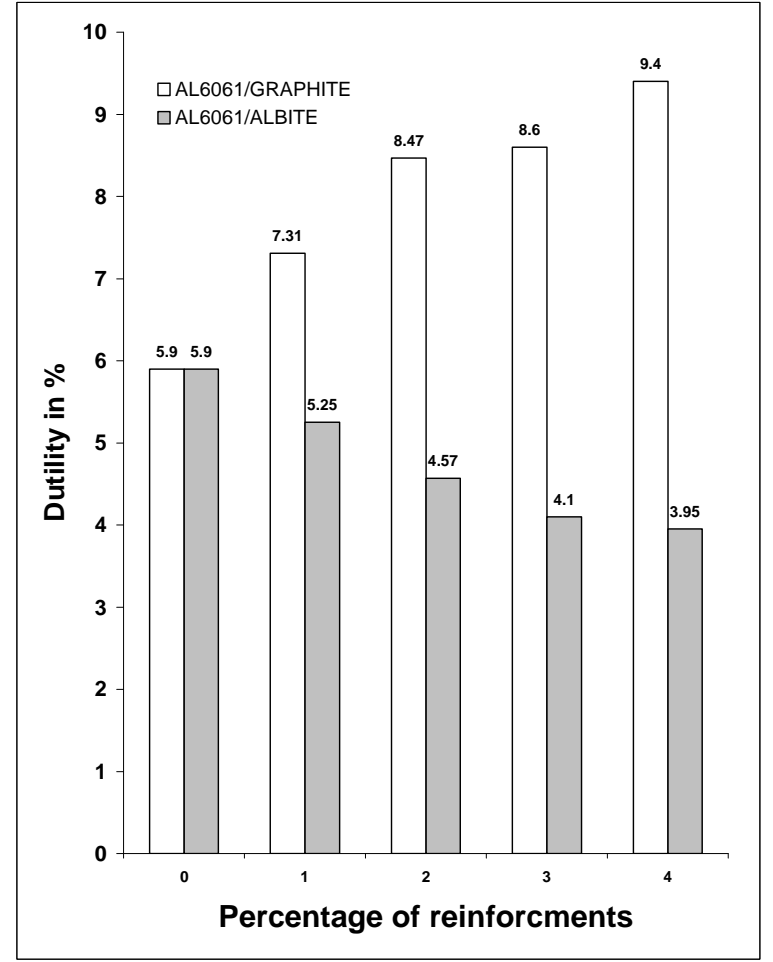

Figure 12. Bar graph of ductility values of AL6061/Graphite and AL6061/Albite metal matrix composites.

Mummery et al. [30] are of the opinion that this loss in ductility is probably due to the voids which nucleate during the plastic straining of the reinforcement. As in the cases of the UTS, hardness and Young's modulus described above, the embrittling effect of albite particulates is expected to be mechanical in nature since the inert albite particulates do not react with the matrix phase [19]. 


\subsection{Compression Strength}

Figure 13 is a graph showing the effect of graphite content on the compressive strength of cast AL6061/graphite particulate composites. As in the cases of ductility and UTS described above, it can be seen that as the graphite content increases, the compressive strength of the composite material increases monotonically by significant amounts. In fact, as the graphite content is increased from $0 \%$ to $4 \%$, the compressive strength increases by about $44 \%$. As in the case of UTS described above, this increase in compressive strength may be due to the graphite particles acting as barriers to dislocations in the microstructure [18]. Once again, this dispersion-strengthening effect is expected to be retained even at elevated temperatures and for extended time periods because the particles are unreactive with the matrix phase [19].

Figure 13 is a graph showing the effect of the albite particulate content on the compressive strength of the composites. It can be seen that the compressive strength of the composite increases monotonically by about, 5 per cent as the albite particulate content is increased from 0 to $4 \mathrm{wt} \%$.

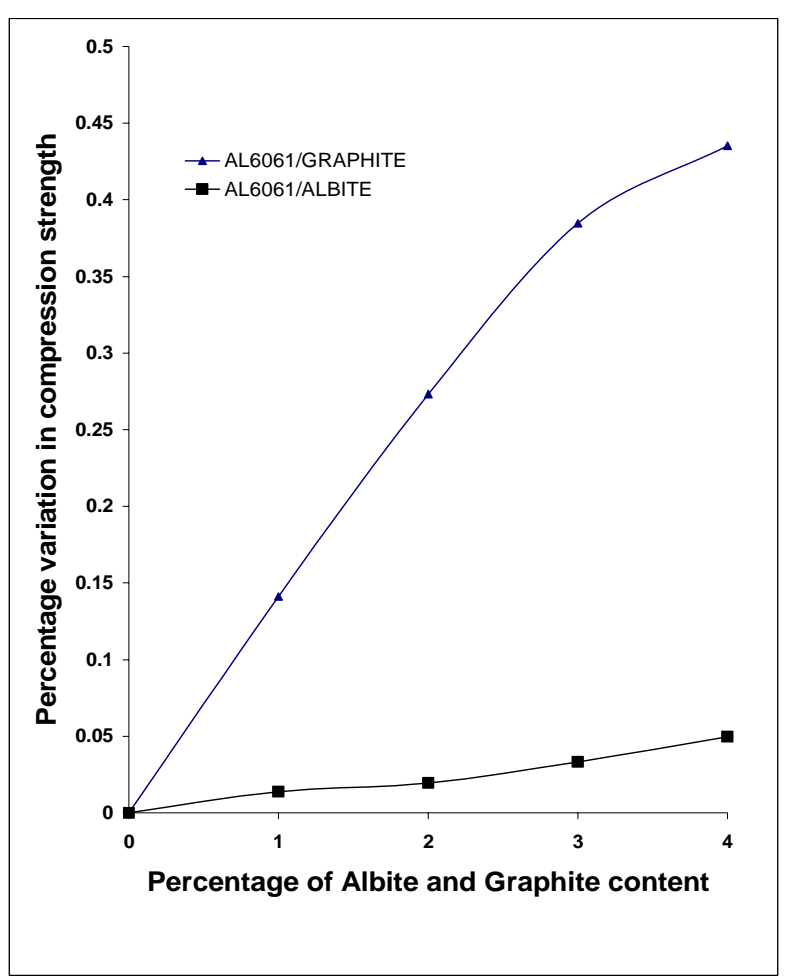

Figure 13. Effect of the albite and graphite content on Ultimate Compression Strength

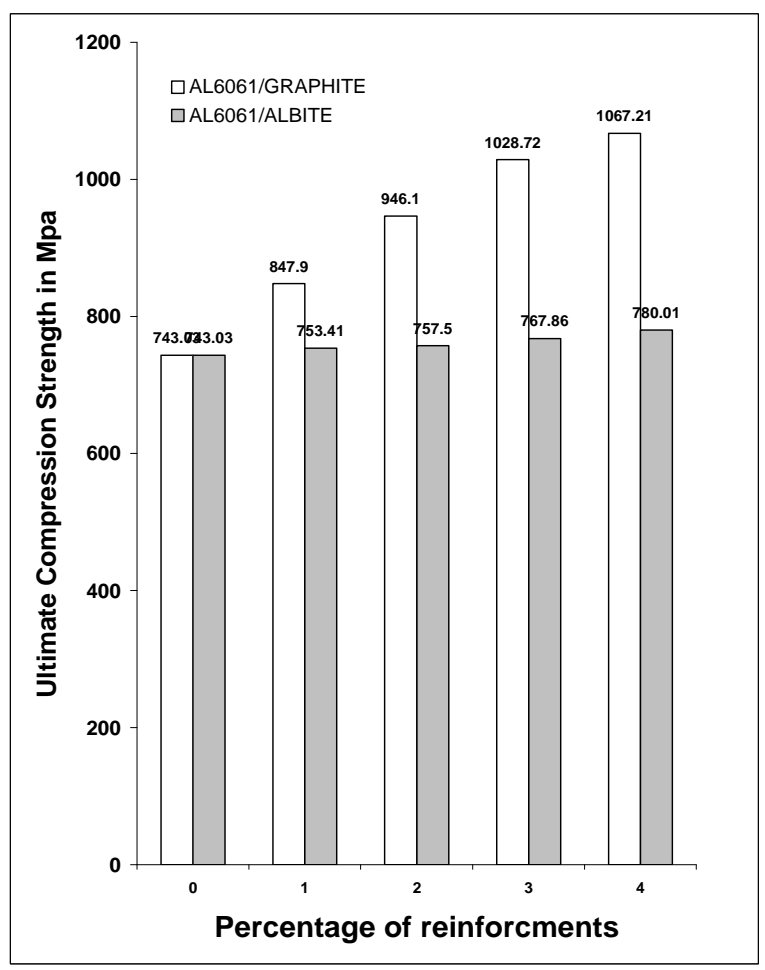

Figure 14. Bar graph of UCS values of AL6061/Graphite and AL6061/Albite metal matrix composites.

Similar results were observed by earlier researchers such as Webster [31] and Awerbuch et al. [13] when they conducted tests on whisker-reinforced composites. Towle and Fried [32] compared the compressive and tensile properties of magnesium-based MMCs and observed similar trends. The increase in compressive strength is chiefly due to the decrease in the interparticle spacing between the albite particles, since albite is much harder than aluminium alloy 6061. The presence of the albite particles resists deforming stresses, thus enhancing the compressive strength of the composite material. The increase in compressive strength is not as spectacular as those seen in the UTS, hardness and Young's modulus because the compressive strength of the unreinforced matrix material itself is already very high, in fact 
several times the UTS. There is only a very marginal increase in the compressive strength when a secondary ceramic phase is introduced. Nevertheless, the addition of hard ceramic particulates has caused the MMCs to behave as brittle rather than ductile materials, as is evident from the above results. The bar graph (Fig 14) shows the comparative changes in UCS, the albite and graphite reinforcements have on the on the matrix alloy AL6061.

\section{CONCLUSIONS}

The mechanical properties of the cast AL6061 /graphite particulate composites are significantly changed by varying the amount of graphite therein. It was found that increasing the graphite content within the aluminum matrix results in significant increases in the ductility, UTS, compressive strength and Young's modulus, but a decrease in the hardness. A compromise is necessary when deciding how much graphite should be added to enhance the mechanical properties of the composite without sacrificing too much of its hardness and hence its wear resistance

The mechanical properties of the cast aluminium alloy 6061-albite particulate composites are significantly altered by varying the amount of albite particulates therein. It was found that increasing the albite particulate content within the aluminium alloy 6061 matrix results in significant increases in the UTS, hardness and Young's modulus but a decrease in the ductility. There is only a slight improvement in its compressive strength. A compromise is necessary when deciding the amount of albite particulates to be added to the aluminium alloy matrix to enhance the UTS, hardness and Young's modulus of the composite without sacrificing too much of its ductility, bearing in mind also that the improvement to its compressive strength is only very marginal

\section{REFERENCES}

[1] Everett, R.K and Arsenault, R.J. Metal Matrix Composites: Mechanisms and Properties, 1991 (Academic Press, San Diego).

[2] Kocjak, M.J., Kahtri, S.C.,Allison, J.E and Jones, J.W. Fundamentals of Metal Matrix Composites (Ed S.Suresh, A.Mortensen and A.Needleman, 1993 (Butterworth-Heinemann, Boston.

[3] Lloyd, D.J. and Brotzen, F.R. Particle reinforced aluminium and Mg matrix composites. Int. Mater.Rev; 1994, 39,1-39.

[4] Lei, M. and Ledbetter, H. Communications: elastic constants of SiCp/Al: measurements and modeling. Metall. Mater. Trans., 1994, 25A, 2832-2835.

[5] Vogelsang, M., Arsenault, R. J. and Fisher, R. M. In-situ HVEM study of dislocation generation at $\mathrm{Al} / \mathrm{SiC}$ interfaces. Metall. Trans. A, 1986, 17, 379.

[6] Pai, B. C., Ray, S., Prabhakar, K. V. and Rohatgi, P. K. Fabrication of aluminium-alumina (magnesia) particulate composites in foundries using magnesium additions to the melts. Mater. Sci. Engng, 1976,24,31.

[7] Sato, A. and Mehrabian, R. Aluminium matrix composites: fabrication and properties. Metall. Trans. B, 1976, 7, 443.

[8] Biswas, S., Santharam, A., Rao, N.A.P, Narayanaswamy, K ., Rohatgi, P. and Biswas, S. K. Tribology International 1980, 8, 171.

[9] Pai, B. C., Pillai, R.M. and Sathyanarayana, K. G, Prospects for graphite aluminium composites in engineering industries. Indian Journal of Engineering and Materials Science 1994, 1, October, 279 
[10] Krishnan, B.P., Raman, N., Narayanaswamy, K and Rohtagi, P.K. Wear, 1980,60,1

[11] Bragg, W. L. Introduction to Crystal Analysis, Bell and Son, London, 1928, p. 64

[12] Pillai, U. T. S., Pandey, R. K. and Nagam, K. D. P. Deformation and fracture of aluminium graphite and aluminium zircon particulate composites. Proc of the 5th International Conference on Composite Materials. TMS Publications, 1985, p. 895

[13] Awerbuch, J., Goering, J. and Busking, K. In Mini Mechanics Analysis and Testing of Short Fibre Composites: Experimental Methods and Results, Vol. 121, 1988. p.964 (American Society for Testing and Materials, Philadelphia. Pennsylvania).

[14] Smith, W. F. Structure and Properties of Engineering Alloys, 2nd edn, McGraw-Hill, New York, 1993, p. 566

[15] Badia, F. A., McDonald, D. F. and Pearson, J. R. Trans. AFS 1971, 79, 269

[16] Biswas, S., Dwarakadasa, E. and Biswas, S. K. Bearings and wear properties of cast graphite aluminium composites. Proc. of All India Seminar on Aluminium, 1979

[17] Pillai, U. T. S. and Pandey, R. K. Studies on mechanical behaviour of cast and forged aluminium graphite particle composites. Journal of Composite Materials 1989, 23, February, 108

[18] Dieter, G. E. Mechanical Metallurgy, SI metric edition, McGrawHill, New York, 1988, pp. 212-19

[19] Callister, W. D. Jr Materials Science and Engineering: An Introduction, 2nd edition, John Wiley, New York, 1991, p. 536.

[20] Ghosh, P. K. and Ray, S. J. Mater. Sci., 1986, 21, 1167.

[21] McCoy, J. M., Prones, C. and Warner, F. E. 1. Mater. Sci., 1988, 1, 37.

[22] Rohatgi, P. K., Ray, S. and Lin, Y. Tribological properties of metal matrix graphite particle composites. International Materials Review, 1992, 37, No.3, 129

[23] K.H.W Seah ., S.C.Sharma., B.M.Girish , Mechanical properties of cast ZA-27/graphite particulate composites, Materials and Design, 1995,16, 271- 275

[24] Zhu, H. X. and Liu, S. K. Mechanical properties of squeeze cast zinc alloy matrix composites containing alpha-alumina fibers. Composites, 1993, 24(6), 437.

[25] Sood, J., Tiwari, A. N. and Teredesai, A. TiC-reinforced Al matrix composites. In Proceedings of Second International Conference on Aluminium INCAL-91, 31 July-2 August 1991, pp. 781-785 (Aluminium Association, Bangalore, India).

[26] Sanders, B. R. and Weintraub, T. L. (Eds) ASM Handbook, Vol. 8, Mechanical Testing, 1995, p. 84 (American Society of Metals, Philadelphia, Pennsylvania).

[27] McDanels, D. L. Analysis of stress-strain, fracture and ductility behaviour of aluminium matrix composites containing discontinuous SiC reinforcement. Metall. Trans. A, 1985, 16, $1105-1115$.

[28] Hoskings, F. M., Portillo, F. F., Wunderlin, R. and Mehrabian, R. Composites of Alalloys: fabrication and wear behavior. J. Mater. Sci., 1982, 17,477--498.

[29] Beitz, W. and Kuttner, K. H. (Eds) Dubbel Handbook of Mechanical Engineering, 1994, p. D8 (Springer-Verlag, London).

[30] Mummery, P. M., Derby, B. and Scruby, C. B. Acoustic emission from particulate reinforced metal matrix composites. Acta Metall., 1993,41,1431.

[31] Webster, D. Metall. Trans. A, 1982, 13A, 511.

[32] Towle, D. J. and Fried, C. M. Comparison and compressive and tensile properties of $\mathrm{Mg}$ based MMCs. J. Mater. Sci. Technol., January 1993, 9, 35--41. 